\title{
Quality of life and satisfaction with life in SLE patients - the importance of clinical manifestations
}

\author{
Lilianna Kulczycka • Anna Sysa-Jędrzejowska • \\ Ewa Robak
}

Received: 7 November 2009/Revised: 27 April 2010/Accepted: 25 May 2010/Published online: 9 June 2010

(C) The Author(s) 2010. This article is published with open access at Springerlink.com

\begin{abstract}
To assess the correlation between quality of life (QoL) and satisfaction with life (SL) in SLE patients and correlate both with clinical symptoms of the disease. The study was performed in 83 patients. QoL was assessed by Short Form 36, and SL was assessed by the Satisfaction with Life Scale. Clinical manifestations presented at the time of examination were taken into consideration. SLE patients assessed their QoL and SL as rather low. Those with photosensitivity as well as neurological symptoms presented lower QoL in particular domains, while those with renal manifestation of SLE assessed their QoL as higher. Similar observations were made for SL only in relation to neurological symptoms. Moreover, our findings show that although SL is a part of QoL, both these parameters should be distinguished in order to fully assess the state of the patient.
\end{abstract}

Keywords Quality of life - Satisfaction with life - SF-36. SWLS $\cdot$ SLE $\cdot$ Clinical manifestations

\section{Introduction}

The term quality of life (QoL) was introduced in the 1970s. Originally, it was restricted to living in particular circumstances, and therefore had an economic value. Later, it was extended to the concept of health and disease. Finally, in the 1990s, Schipper [1-3] proposed the notion of healthrelated quality of life (HRQoL). This expression is

L. Kulczycka $(\bowtie) \cdot$ A. Sysa-Jędrzejowska $\cdot$ E. Robak

Department of Dermatology and Venereology,

Medical University of Lodz,

Krzemieniecka Street No 5,

94-017 Lodz, Poland

e-mail: lilakulczycka@wp.pl narrower than QoL because HRQoL only focuses on the influence of health and disease $[4,5]$. It is often used for chronic and incurable diseases, without taking other nonmedical aspects into account.

While assessing QoL, the satisfaction with life (SL) is also considered. The term SL is very similar to quality of life, nevertheless the terms should be distinguished; QoL is affected by more aspects than SL and includes other issues such as age, sex, education, occupation, social functioning, and of course, health, whereas satisfaction with life can be understood as contentment, and is a part of the subjective well-being connected with happiness and positive emotions [6]. Furthermore, satisfaction with life is defined as a difference between one's own assumptions and plans, and real achievements [7]. However for some researchers, the two terms are indistinguishable. Our aim, therefore, was not to distinguish those two terms, but to compare them or discover whether the influence of a disease's clinical symptoms, and of other factors connected with its course, has a significant effect on QoL and SL.

Systemic lupus erythematosus (SLE) is a chronic disease of the connective tissues, which can wax and wane [8]. It occurs ten times more frequently in women, more so in young ones. Because of the vast diversity of clinical manifestations and damage to organs which occur in the course of the disease, the predictions are uncertain.

To fully assess the state of patients, especially those with chronic and incurable diseases such as SLE, quality of life is frequently taken into consideration [9] and a QoL assessment is performed to recognize and understand any determining factors. The next step is an attempt to improve QoL. To our knowledge, even though there are numerous publications concerning quality of life and systemic lupus erythematosus, the influence of clinical symptoms on patients' QoL is not studied enough. Moreover, there is a 
paucity of reports which consider satisfaction of life in SLE as well as its associations with QoL.

\begin{abstract}
Aim
The main aim of this paper is to assess quality of life, together with satisfaction with life, in systemic lupus erythematosus patients. Moreover, we will attempt to answer the question whether the patients who assess their own QoL as high will also evaluate their SL in the same way. Any correlations between those parameters and clinical manifestation of the disease, activity of the disease, and damage index will also be noted.
\end{abstract}

\section{Materials and methods}

The study was performed on 83 patients (78 women and five men) under the care of the Department of Dermatology and Venereology, Medical University of Lodz as well as its Outpatient Clinic. The average duration of the disease was about 8.9 years. SLE activity was assessed using Systemic Lupus Activity Measure (SLAM) [10]. The disease was assumed to be active when the patient scored 7 or more points [11]. The degree of damage which could occur during the course of SLE was determined on the basis of the Systemic Lupus International Collaborating Clinics/ American College of Rheumatology Damage Index [12].

Most of the patients (59\%) achieved 7 or more points in SLAM, indicating that SLE symptoms were clinically important [11]. Nevertheless, they presented a lowdamage index. Among the most often observed clinical manifestations of SLE, are: malar rush, photosensitivity, arthralgia, and laboratory abnormalities (anemia, leucopenia, or thrombocytopenia). Clinical characteristics of the patients are presented in Table 1.

The quality-of-life measurement was made using the Short Form 36 Health Survey (SF-36) [13]. This is a standard questionnaire that allows quality of life to be assessed, both in the general healthy population as well as groups of sick people. It consists of 36 questions grouped into 8 domains measuring different aspects of QoL (Physical Functioning (PF), Role Physical (RP), Bodily Pain (BP), General Health (GH), Vitality (VT), Social Functioning (SF), Role Emotional (RE), Mental Health (MH)). Obtained results are converted into a scale from 0 to 100 , where 0 means the lowest quality of life and 100 means the highest one, which makes it possible to interpret data and draw conclusions about the patients' quality of life without using norms (which do not exist for Polish population) or a control group.

The Satisfaction with Life Scale was used to measure the patients' satisfaction with life [14]. It evaluates satisfaction
Table 1 Characteristics of the patients

\begin{tabular}{ll}
\hline Parameter & Value \\
\hline Number of patients & 83 \\
Women/men & $78 / 5$ \\
Mean age (range) & $42.7(21-71)$ years \\
Mean duration of the disease & 8.9 years \\
Activity of the disease (SLAM) & $4-23(x=12.31)$ \\
Patients in an inactive stage of SLE & $34(41 \%)$ \\
Patients in an active stage of SLE & $49(59 \%)$ \\
Clinical manifestations & \\
Malar rush & $43(52 \%)$ \\
Discoid rash & $6(7 \%)$ \\
Photosensitivity & $64(77 \%)$ \\
Oral ulcers & $6(7 \%)$ \\
Arthralgia & $73(88 \%)$ \\
Serositis & $5(6 \%)$ \\
Renal disorder & $18(22 \%)$ \\
Neurologic disorder & $10(12 \%)$ \\
Hematologic disorder & $55(66 \%)$ \\
Immunologic disorder (anti-dsDNA, anti-Sm) & $13(16 \%)$ \\
SLICC/ACR DI & \\
Patients who obtained 0 point & $50(60.24 \%)$ \\
Patients who obtained $>1$ point & $33(39.76 \%)$ \\
Damage index-medium; SD & $\mathrm{x}=0.67 ; \mathrm{SD}=1.11$ \\
Damages: & \\
Ocular & $6(18.18 \%)$ \\
Neuropsychiatric & $10(30.3 \%)$ \\
Renal & $5(15.15 \%)$ \\
Pulmonary & 0 \\
Cardiovascular & $7(21.21 \%)$ \\
Peripheral vascular & $7(21.21 \%)$ \\
Gastrointestinal & \\
Mucoskeletal & \\
Skin & $(12.12 \%)$ \\
Other & \\
\hline & \\
& \\
\hline
\end{tabular}

with life without taking into consideration the somatic symptoms of the disease, and is suitable for people at any age. The questionnaire consists of five statements to which the patient assigns a value from 1 to 7 , depending on how much the respondent agrees with it. It is possible to get a total score between 5 to 35 points. The scores are then converted into stens from 0 to 10 . A sten is a unit of a psychological test which is normalized so that the mean value of the population is 5.5 , and the standard deviation is 2 . According to the Polish norms, a result from 1 to 4 stens reflects low satisfaction with life, 5 and 6 medium, and from 7 to 10 high.

The study was approved by the Bioethics Committee of the Medical University of Lodz, Poland (Number RNN/ 124/06/KE). The patients were informed about the aim of 
the study and all of them gave informed consent for participation.

\section{Statistical analysis}

All results were presented as numeral values from minimum (Min) to maximum (Max), also the arithmetical mean $(x)$, median (Me), and standard deviation (SD) were given. Mean results were compared by the Mann-Whitney $U$ and the Kruskal-Wallis tests. Correlations between the obtained results were assessed using the Spearman's rank correlation coefficient $(\rho)$. Differences at $p<0.05$ were considered statistically significant.

\section{Results}

Quality of life and satisfaction with life

The obtained data revealed that the patients with systemic lupus erythematosus presented rather low scores in both quality of life as well as satisfaction with life; they did not achieve mean values of 60 in any QoL domain (Fig. 1). Particularly low results were observed in two domains: RP $(x=8.66, \mathrm{SD}=11.83)$ and $\mathrm{RE}(x=11.55, \mathrm{SD}=10.64)$, while other parameters that assessed psychological and somatic functioning were also low, but still relatively higher (Fig. 2). The patients obtained the highest results in three domains: $\mathrm{PF}(x=55.36, \mathrm{SD}=26.64), \mathrm{SF}(x=56.48, \mathrm{SD}=$ $27.49)$, and $\mathrm{MH}(x=51.76, \mathrm{SD}=17.64)$. Most of the patients $(62 \%)$ assessed their satisfaction with life as low (0-4 stens), $17 \%$ of the patients assessed it as medium (5-6 stens), and $21 \%$ as high ( $7-10$ stens).
In the range of low values, the patients gave answers that were close to the medium results. A similar situation was observed for medium values, but not for high ones. Here, the answers were close to the lower border (Fig. 3).

Looking for correlations between quality of life and satisfaction with life, statistically significant positive correlations were observed for all SF-36 domains (Table 2).

Quality of life and clinical manifestations of SLE

The patients presented a vast diversity of clinical symptoms for SLE. The most common manifestations were arthralgia, myalgia, photosensitivity, malar rush, and hematological abnormalities (Table 1). Statistically significant negative correlations were observed between photosensitivity and $\mathrm{RE}(p=0.04)$ as well as between neurological symptoms of SLE and SF ( $p=0.04)$ (Fig. 3). For renal manifestations of the disease and $\mathrm{MH}$, the observed correlations were also statistically significant but positive $(p=0.04)$ (Fig. 3). There were no statistically significant correlations between other clinical symptoms of SLE and quality of life $(p>0.05)$.

Satisfaction with life and clinical manifestations of SLE

A statistically significant negative correlation between neurological symptoms and SL was present $(p=0.04)$. Correlations for other manifestations were statistically insignificant $(p>0.05)$.

Quality of life, satisfaction with life, and activity of the disease

Statistically significant negative correlations were found only between the activity of SLE and PF, MH and VT
Fig. 1 Quality of life in SLE patients using SF-36

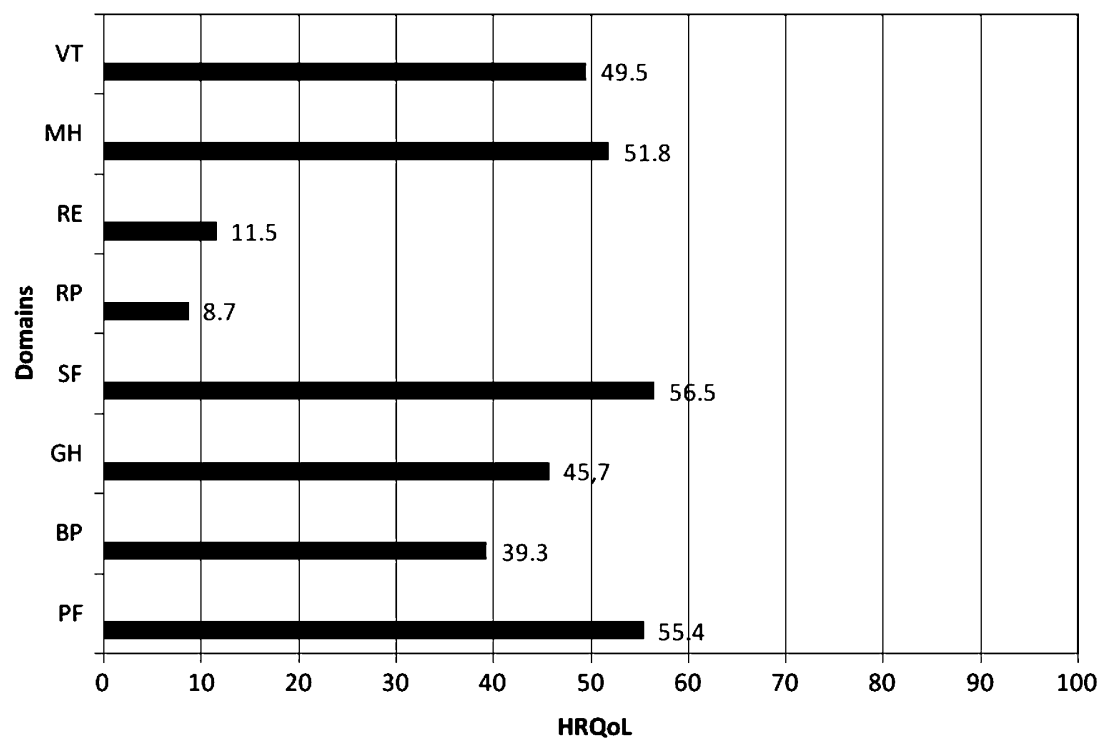




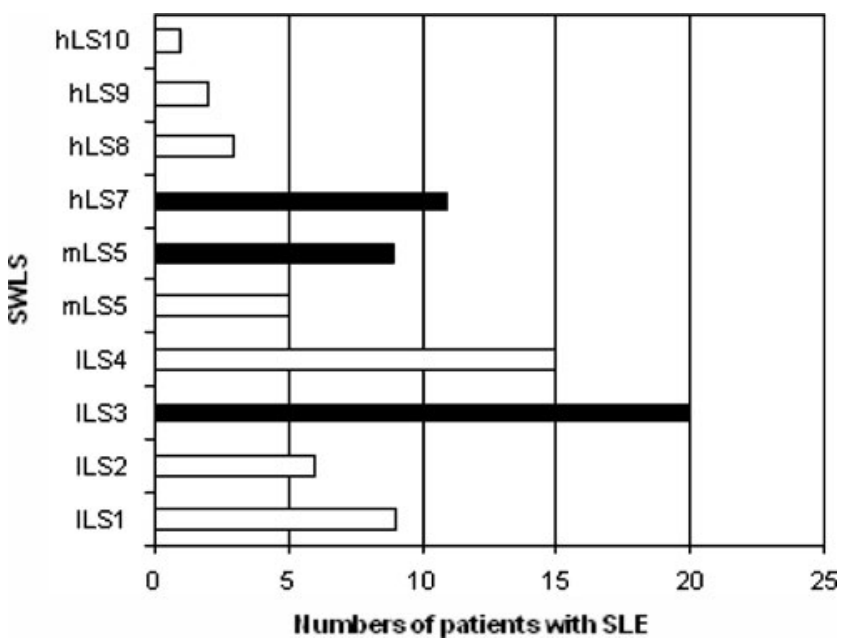

Fig. 2 Percentage distribution of satisfaction with life in SLE patients (lLS1-lLS4 low life satisfaction, $m L S 5-m L S 6$ medium life satisfaction, $h L S 7-h L S 10$ high life satisfaction). The most common answers in all three divisions of satisfaction are marked in black

(Table 3). For MH, the correlation was on the border of statistical significance. There were no statistically significant correlations between the activity of the disease and other domains of SF-36 (Table 3).

In the investigated group of the patients, a negative but statistically insignificant correlation was present between the satisfaction with life and the activity of the disease $(p>0.05)$.

The results obtained by the SLE patients in an active and inactive state are presented in Table 4.

Quality of life, satisfaction with life, and damage index

Statistically significant negative correlations were present only between the damage index and RP as well as VT (Table 5). There were no statistically significant correlations between the damage index, the satisfaction with life and the activity of SLE $(p>0.05)$.

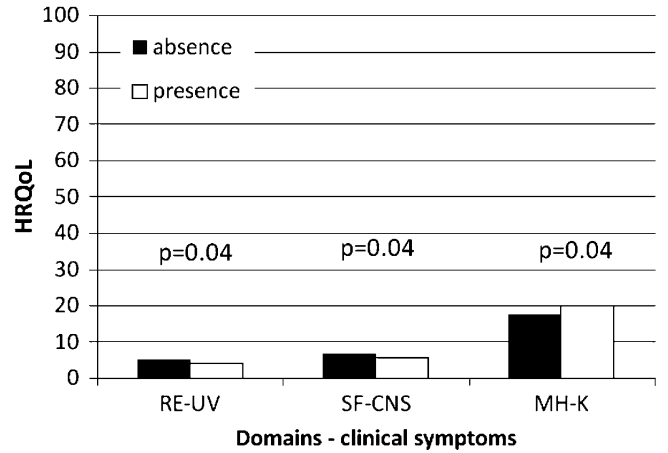

Fig. 3 Correlations between domains of SF-36 and clinical manifestations of SLE. $R E$ role emotional, $U V$ photosensitivity, $S F$ social functioning, $C N S$ neurological symptoms of SLE, $M H$ mental health, $K$ renal manifestations of SLE
Table 2 Correlations between quality of life using SF-36 and satisfaction with life using SWLS

\begin{tabular}{lll}
\hline SWLS vs & $\rho^{\mathrm{a}}$ & $p^{\mathrm{b}}$ \\
\hline PF & 0.43 & $<0.001$ \\
BP & 0.37 & $=0.001$ \\
GH & 0.42 & $<0.001$ \\
SF & 0.51 & $<0.001$ \\
RP & 0.31 & $=0.006$ \\
RE & 0.67 & $<0.001$ \\
MH & 0.57 & $<0.001$ \\
VT & 0.44 & $<0.001$
\end{tabular}

${ }^{a}$ Spearman's rank correlation coefficient,

${ }^{\mathrm{b}}$ Statistical significance

\section{Discussion}

Our results reveal that systemic lupus erythematosus, as a chronic and incurable disease, has a great influence on the patients' quality of life as well as their satisfaction with life. Moreover, differences between the presented results of QoL and SL suggest that those two terms are slightly different but still complementary to each other. The investigated group of the patients assessed their quality of life as medium and indicated that the disease had the highest influence on the RP and RE. BP was also quite important. Nevertheless, the patients assessed their PF, and VT as high in comparison with other QoL domains. In the literature, there is agreement that systemic lupus erythematosus induces a lower quality of life in patients [15-21]. Differences in particular domains of SF-36 are mainly caused by cultural, socio-economical, and clinical factors. Results which reveal the impact of those factors on the patients' quality of life, as well as particular domains measured in SF-36, were presented elsewhere [22]. The factors taken into consideration in this study were the level of education,

Table 3 Correlations between activity of SLE (using SLAM) and quality of life (using SF-36)

\begin{tabular}{lll}
\hline Activity of SLE vs & $\rho^{\mathrm{a}}$ & $p^{\mathrm{b}}$ \\
\hline PF & -0.31 & $=0.02$ \\
BP & -0.25 & $>0.05$ \\
GH & -0.07 & $>0.05$ \\
SF & -0.21 & $>0.05$ \\
RP & -0.20 & $>0.05$ \\
RE & -0.19 & $>0.05$ \\
MH & -0.25 & $=0.05$ \\
VT & -0.32 & $=0.03$ \\
\hline
\end{tabular}

${ }^{\text {a }}$ Spearman's rank correlation coefficient

${ }^{\mathrm{b}}$ Statistical significance 
Table 4 Results of satisfaction with life in SLE patients depends on activity of the disease

\begin{tabular}{lllll}
\hline & $x^{\mathrm{a}}$ & Min & Max & SD \\
\hline Satisfaction with life/active SLE & 16.45 & 6.0 & 33.0 & 6.92 \\
Satisfaction with life/inactive SLE & 15.56 & 7.0 & 27.0 & 5.40 \\
\hline
\end{tabular}

Min minimal values, Max maximal values, $S D$ standard deviation

${ }^{\mathrm{a}}$ Mean values

social conditions, source of money, and information whether the patient was the only person working in the family. To summarise, we showed that worse socioeconomic conditions were correlated with a higher satisfaction with life.

There are not many reports in the literature on the satisfaction with life of chronically ill patients. Moreover, according to our knowledge, no studies on this subject have been published in relation to patients with connective tissue diseases, especially SLE. A Polish study carried out by Juczyński et al. [14] apart from presenting the results of SL assessed by the Satisfaction with Life Scale in patients with selected chronic diseases (diabetes, stage renal disease (ESRD), and status post myocardial infarction) also contains the Polish norms.

Our results have revealed that the patients registered a low satisfaction with life around twice as often as compared with the general Polish population. They have a similar relationship to the results achieved for quality of life, which was further confirmed by statistically significant correlations between QoL and SL. Therefore, it can be seen that when quality of life increases, satisfaction is also higher. This relationship provides evidence that satisfaction is a part of quality of life. Results obtained by SLE patients are similar to those of patients with ESRD as well as men after myocardial infarction [14].

On the other hand, the presented differences indicate that those two terms are probably separate. It seems that quality of life is a wider notion that remains closely related not only to the somatic state of the patient but also to other factors, such as socioeconomic status and demographic factors. It therefore contains elements which can have a long-lasting impact on it. On the other hand, satisfaction with life seems to be related to emotions, expectations or even attitude towards some events, thus it describes the state which is more variable. Obviously, it is also somehow connected with other factors such as age, sex, education, or social functioning. The patient might report a low quality of life because of a chronic disease which leads to disability, while satisfaction with life is high because the patient can still work or is satisfied with his/her family life. It seems that a strict and precise separation of these two terms is not possible. The investigated group of patients consisted of people who lived in good social conditions. They were mainly working or they received a disability pension. Although we have revealed that patients' better living conditions are connected with higher satisfaction with life (unpublished results), this is the opposite of the relationship between their living conditions and quality of life, in that better living conditions correlate with lower QoL [22]. It can confirm the hypothesis that both terms should be measured separately, as they cover slightly different areas of life.

Literature data indicates that even if symptoms of the disease are not very intensive and activity of the disease is rather low, they can have a huge influence on quality of life. In the present research, correlations between photosensitivity and the RE, neurological symptoms of SLE and SF, as well as renal symptoms and MH were found. For other clinical manifestations of SLE and domains of SF-36, no correlations were present. It should be emphasized that the patients studied had a mild or moderate course of the disease. There were no patients with a severe renal involvement, or with advanced neurological symptoms. Further research is definitely needed in this field, ideally conducted among patients with a severe course of the disease, to allow a more comprehensive assessment of the quality of life, as well as satisfaction with life.

In the literature, there is no information on the relationship between the clinical symptoms of the disease and satisfaction with life among SLE patients. In our group, only patients with a neurological manifestation of the disease presented lower life satisfaction, and lower quality of life, whereas no correlations between SL and renal involvement or photosensitivity were found. This observation suggests that quality of life and satisfaction with life are close terms.

The results of various studies differ as regards the influence of SLE activity on patients' quality of life $[18,21$, 23-26], which might be due to using different activity

Table 5 Correlations between damage index (DI) and quality of life in SLE patients (using SF-36)

\begin{tabular}{lll}
\hline DI vs & $\rho^{\mathrm{a}}$ & $p^{\mathrm{b}}$ \\
\hline PF & -0.13 & $>0.05$ \\
BP & -0.01 & $>0.05$ \\
GH & 0.07 & $>0.05$ \\
SF & -0.01 & $>0.05$ \\
RP & -0.23 & $=0.04$ \\
RE & -0.14 & $>0.05$ \\
MH & -0.06 & $>0.05$ \\
VT & -0.22 & $=0.04$
\end{tabular}

${ }^{\text {a }}$ Spearman's rank correlation coefficient

${ }^{\mathrm{b}}$ Statistical significance 
scales as well as a dissimilar number of patients, diverse clinical manifestation of the disease, and also socioeconomic and cultural factors. The present study has indicated that when the disease activity was higher, the patients reported a lower quality of life. However, similar findings were not observed for satisfaction with life. This suggests that QoL and SL may be assessed separately to enable a full examination of the patient's state.

Also, the damage index (DI) and its effect on quality of life and satisfaction with life seem to play an important role. We revealed that the damage to organs occurring during the disease course resulted in lower RP and VT although no similar correlation between DI, activity of the disease or SL was found. This observation might to a certain degree confirm some differences between quality of life and satisfaction with life. Moreover, it also suggests that a clinical state of the patient assessed by measuring the disease activity and damage does not go together with SL, but at the same time creates QoL. According to the literature data [27], it seems that results of SF-36 do not fluctuate over time together with an increase in disease activity and damage index. However, it is not known whether the same situation pertains to satisfaction with life. We believe that a longitudinal design is needed. It is worth underlining once more that only patients with an unadvanced course of the disease participated in the study, which may well have had an influence on the final result.

Many authors have been interested in the problem of the effect of the damage index on quality of life in SLE patients. Results presented by them confirmed statistically significant correlations between domains of SF-36 and DI $[15,21,24,28-30]$. It seems that not the numerical value of DI but the localization of damage is most important for the future of the patient. Some authors $[26,31]$ consider that the damage index affects only a mental aspect of QoL: a consequence of coping strategies. The presented results are in opposition to the observations made by other authors $[16,19,24,32]$ who did not find any correlations between the damage index and the quality of life in SLE patients. The results presented by Gilboe et al. are interesting [27]. They observed patients for 2 years and showed that their quality of life as well as activity of the disease did not change although the damage index was higher. The authors underlined the lack of changes in quality of life, although this may have been due to the patients becoming accustomed to the disease, accepting it, and finding their own way to deal with it.

The results presented in this study have proven that systemic lupus erythematosus has a great influence on the psyche of the patients and indicate that there is a huge need to support them. However, a longitudinal study might show the aforementioned correlations over time. It seems that standard therapeutic management should consist of meas- urements of quality of life, and also satisfaction with life, together with a clinical assessment of the patient's state as well as activity of the disease, collectively with the damage index Even if clinical symptoms of SLE are not very noticeable to the doctor, they may be a problem for the patient and they cannot be ignored. The awareness that the disease has such a strong impact on a mental state of the patient as well as attitude towards life may improve compliance.

The study was completed by measuring satisfaction with life. Our results reveal that there are differences between quality of life and satisfaction with life but also underlined the importance of both aspects. Therefore, it is reasonable to continue research and undertake an effort to improve the mental comfort of the patients. We do believe that further investigations in this field are necessary, especially with a group of patients with more severe signs and symptoms of SLE. It will allow a more precise assessment of the effect of the disease on both quality of life and satisfaction with life.

Acknowledgements The study was financially supported by grants No 502-11-727 and 503-1152-1 from the Medical University of Lodz. The authors thank Ms Elżbieta Dziankowska-Zaborszczyk for performing statistical analysis of the data.

\section{Disclosures None.}

Open Access This article is distributed under the terms of the Creative Commons Attribution Noncommercial License which permits any noncommercial use, distribution, and reproduction in any medium, provided the original author(s) and source are credited.

\section{References}

1. Felce D, Perry J (1995) Quality of life: it's definition and measurement. Res Dev Disabil 16:51-74

2. Dziurowicz-Kozłowska A (2002) Wokół pojęcia jakości życia. Psychologia Jakości Życia 1:77-99

3. Schipper H (1990) Quality of life: principles of the clinical paradigm. J Psychol Oncol 8:171-185

4. Guyatt GH, Feeny DH, Patrick DL (1993) Measuring healthrelated quality of life. Ann Int Med 118:622-629

5. Muldoon MF, Barger SD, Manuck SB (1998) What are quality of life measurements measuring? BMJ 316:542-545

6. Diener E (1984) Subjective well-being. Psychol Bull 95:542-575

7. Bowling A (1997) Measuring health. Open University Press Buckingham, Philadelphia

8. Mills JA (1994) Systemic lupus erythematosus. N Eng J Med 330:1871-1879

9. Gladman DD (1995) Prognosis and treatment of systemic lupus erythematosus. Curr Opin Rheumatol 7:402-408

10. Liang MH, Socher SA, Larson MG et al (1989) Reliability and validity of six system for the clinical assessment of disease activity in systemic lupus erythematosus. Arthritis Rheumatol 7:817-823

11. Abrahamowicz M, Fortin PR, Du BR et al (1998) The relationship between disease activity and expert physician's decision to start major treatment in active systemic lupus erythematosus: a decision 
aid for development of entry criteria for clinical trials. J Rheumatol 25:277-284

12. Griffiths B, Mosca M, Gordon C (2005) Assessment of patients with systemic lupus erythematosus and the use of lupus disease activity index. Best Pract Res Clin Rheumatol 19:685-708

13. Ware JE, Kosinski M, Dewey JE (2000) How to score version 2 of the SF-36 ${ }^{\circledR}$ Health Survey. QualityMetric Incorporated, Lincoln, RI

14. Juczyński Z (2001) Narzędzia pomiaru w promocji i psychologii zdrowia, Pracownia Testów Psychologicznych Polskiego Towarzystwa Psychologicznego, Warszawa

15. Stoll T, Gordon C, Seifert B et al (1997) Consistency and validity of patient administrated assessment of quality of life by MOS SF36; its association with disease activity and damage in patients with systemic lupus erythematosus. J Rheumatol 24:1608-1614

16. Gladman DD, Urowitz MB, Gough J et al (1997) Fibromyalgia is a major contributor to quality of life in lupus. J Rheumatol 24:2145-2148

17. Stoll T (2001) Prediction of depression in systemic lupus erythematosus patients using SF-26 Mental Health Scores. Rheumatology 40:695-698

18. Benitha R, Tikly M (2007) Functional disability and health-related quality of life in South Africans with rheumatoid arthritis and systemic lupus erythematosus. Clin Rheumatol 26:24-29

19. Freire EA, Maia IO, Nepomuceno JC et al (2007) Damage index assessment and quality of life in systemic lupus erythematosus patients (with long term disease) in Northeastern Brazil. Clin Rheumatol 26:423-428

20. Rinaldi S, Doria A, Salaffi F et al (2004) Health-related quality of life in Italian patients with systemic lupus erythematosus. I. Relationship between physical and mental dimension and impact of age. Rheumatol 43:1574-1579

21. Alarcon GS, McGwin G, Uribe A et al (2004) Systemic lupus erythematosus in a multiethnic lupus cohort (LUMINA). XVII. Predictors of self-reported health-related quality of life early in disease course. Arthritis Rheumatol 51:465-474
22. Kulczycka L, Sysa-Jędrzejowska A, Zalewska-Janowska A et al (2008) Quality of life and socioeconomic factors in Polish patients with systemic lupus erythematosus. JEADV 22:1218-1226

23. Saba J, Quinet RJ, Davis WE et al (2003) Inverse correlation of each functional status scale of the SF-36 with degree of disease activity in systemic lupus erythematosus (m-SLAM). Jt Bone Spine 70:348-351

24. Vu TV, Escalante A (1999) A comparison of the quality of life of patients with systemic lupus erythematosus with and without endstage renal disease. J Rheumatol 26:2595-2601

25. Gladman DD, Urowitz MB, Ong A et al (1996) Lack of correlation among 3 outcomes describing SLE: disease activity, damage and quality of life. Clin Exp Rheumatol 14:305-308

26. Dobkin P, da Costa D, Drista M et al (1999) Quality of life in systemic lupus erythematosus patients during more and less active disease states: differential contributors to mental and physical health. Arthritis Care Res 12:401-410

27. Gilboe IM, Kvien TK, Husby G (2001) Disease course in systemic lupus erythematosus: changes in health status, disease activity, and organ damage after 2 years. J Rheumatol 28:266-274

28. Doria A, Rinaldi S, Ermani M et al (2004) Health-related quality of life in Italian patients with systemic lupus erythematosus. II. Role of clinical, immunological and psychological determinants. Rheumatol 43:1580-1586

29. Wang C, Mayo NE, Fortin PR (2001) The relationship between health related quality of life and disease activity and damage in systemic lupus erythematosus. J Rheumatol 28:525-532

30. Sutcliffe N, Clarke AE, Levinton C et al (1999) Associates of health status in patients with systemic lupus erythematosus. J Rheumatol 26:2352-2356

31. Thumboo J, Fong KY, Chan SP et al (2000) A prospective study of factors affecting quality of life I systemic lupus erythematosus. J Rheumatol 27:1414-1420

32. Thumboo J, Fong KY, Ng TP et al (1999) Validation of the MOS SF-36 for quality of life of patients with systemic lupus erythematosus in Singapore. J Rheumatol 26:96-102 